\title{
Bile canalicular abnormalities in the early phase of a mouse model of sclerosing cholangitis.
}

\section{AUTHOR(S):}

Miyao, Masashi; Ozeki, Munetaka; Abiru, Hitoshi; Manabe, Sho; Kotani, Hirokazu; Tsuruyama, Tatsuaki; Tamaki, Keiji

\section{CITATION:}

Miyao, Masashi ...[et al]. Bile canalicular abnormalities in the early phase of a mouse model of sclerosing cholangitis.. Digestive and liver disease 2013, 45(3): 216-225

\section{ISSUE DATE:}

2013-03

URL:

http://hdl.handle.net/2433/173350

\section{RIGHT:}

(c) 2012 Editrice Gastroenterologica Italiana S.r.l. Published by Elsevier Ltd.; This is not the published version. Please cite only the published version.; この論文は出版社版でありません。引用の際には出版社版を ご確認ご利用ください。 
Bile canalicular abnormalities in the early phase of a mouse model of sclerosing cholangitis

Masashi Miyao ${ }^{1}$ M.D.; Munetaka Ozeki ${ }^{1}$ Ph.D.; Hitoshi Abiru ${ }^{1}$ B.S.; Sho Manabe ${ }^{1}$ B.S.; Hirokazu Kotani ${ }^{1}$ M.D.; Tatsuaki Tsuruyama ${ }^{2}$ M.D. and Keiji Tamaki ${ }^{1}$ M.D.

${ }^{1}$ Department of Forensic Medicine and Molecular Pathology, Kyoto University Graduate School of Medicine

${ }^{2}$ Center for Anatomical Studies, Kyoto University Graduate School of Medicine

\section{Corresponding author:}

Keiji Tamaki. Telephone: +8175753 4474. Fax: +81 75761 9591. E-mail:

ktamaki@fp.med.kyoto-u.ac.jp. Department of Forensic Medicine and Molecular Pathology, Kyoto University Graduate School of Medicine, Yoshida-Konoe-cho, Sakyo-ku, Kyoto 606-8501, Japan

Conflict of Interest: The authors declare no conflict of interest.

Word count: 3484 words (excluding abstract, references, figures). 


\section{Abstract}

Background: The bile canaliculus is the smallest and first biliary channel and is formed by two or three adjacent hepatocytes. Previous studies of chronic cholangiopathies such as primary sclerosing cholangitis have focused on the bile ductules. However, little is known about the pathological alterations in bile canaliculi in the early phase of cholangiopathies.

Aim: To characterize the bile canalicular morphology in the early phase of sclerosing cholangitis we used 3,5-diethoxycarbonyl-1,4-dihydrocollidine-induced mouse model of sclerosing cholangitis.

Methods: Mice were fed a diet with 3,5-diethoxycarbonyl-1,4-dihydrocollidine (0.1\%). Serum biochemical, histological, immunohistochemical, and electron microscopic analyses were performed 1, 2, 4, and 7 days after feeding.

Results: All experimental groups showed significantly increased serum aspartate aminotransferase, alanine aminotransferase, and alkaline phosphatase levels. From day 1, bile canalicular abnormalities such as dilatation and meandering and loss of microvilli were observed. After bile canalicular abnormalities had appeared, substantial infiltration of inflammatory cells was observed amongst the necrotic cells and periductal region. After these inflammatory changes, cholangiocytes proliferated in the portal area and formed ductular reactions. Finally, periductal fibrosis appeared.

Conclusion: This study provides novel evidence of the occurrence of bile canalicular 
abnormalities during the early phase of sclerosing cholangitis.

Keywords: Bile canaliculus; Cholangiopathy; 3,5-diethoxycarbonyl-1,4-dihydrocollidine;

Ductular reactions 


\section{Introduction}

The bile canaliculus is the smallest and first biliary channel and is formed by two or three adjacent hepatocytes [1]. Bile canaliculi are located in the liver parenchyma and directly receive bile secreted by the hepatocytes. The bile canaliculi are exposed to and injured by toxic bile acids before the cholangiocytes in the bile ductules located in the portal area are injured by these bile acids. However, little is known about the pathological alterations of the bile canaliculi during the early phase of cholangiopathies. Bile is a complex solution consisting of inorganic and organic compounds including bile acids [2-4]. Even in normal conditions, bile acids are considerably toxic and directly induce cholangiocyte injury. Cholangiocytes construct bile ducts such as Hering's canals, bile ductules, and intra- and extra-hepatic biliary ducts. Previous reports have demonstrated that bile injures cholangiocytes during its drainage through these ducts from the liver parenchyma to the duodenum, leading to chronic cholangiopathies [5-6].

Chronic cholangiopathies such as primary sclerosing cholangitis (PSC) and primary biliary cirrhosis (PBC) are characterized by chronic bile duct injury. In previous reports, the cholangiocytes of the bile ducts have been assumed to play a central role in the pathogenesis of these diseases as a primary target and also by inducing inflammation and fibrosis [7]. Cholangiocytes are injured by hydrophobic bile acids because the bile acids are directly toxic to cholangiocytes [8]. In addition, cholangiocytes that are activated (reactive cholangiocytes) 
by toxic bile acids release proinflammatory cytokines, which then activate neutrophils, Kupffer cells, T cells, and natural killer cells. Simultaneously, activated cholangiocytes proliferate, leading to increased numbers of bile ductules. These reactions of cholangiocytes are referred to as ductular reactions (reactive cholangiocyte phenotype) [5, 9]. During the chronic phase, reactive cholangiocytes play a role in reparative and fibrogenic processes, leading to periductal fibrosis and eventually, biliary cirrhosis. During this phase, they release profibrogenic cytokines that affect hepatic stellate cells and portal fibroblasts, which differentiate into myofibroblasts that produce excess amounts of collagen fibers and extracellular matrix. Thus, previous studies have proposed that ductular reactions and portal fibrosis play a central role in cholangiopathies, but the role of bile canaliculi in the parenchyma remains unknown.

Most data regarding the pathogenesis of cholangiopathies are derived from in vivo studies using animal models such as bile duct-ligated or carbon tetrachloride-treated rodents [10-11]. These models, however, are unsuitable for studying the early phase of cholangiopathies because the animals deteriorate rapidly, and the reproducibility of these models is low. To explore the pathological changes in bile canaliculi during the earlier phase, a gradually progressing cholangiopathy model is needed. Recently, Fickert et al. demonstrated that continuous feeding of mice with 3,5-diethoxycarbonyl-1,4-dihydrocollidine (DDC) induces chronic cholangiopathy with an indolent course. This model is well established for studying 
the mechanisms of sclerosing cholangitis [12] and is expected to be suitable for investigating the precise events that occur during the early phase of cholangiopathies including sclerosing cholangitis.

Most patients with cholangiopathies display a progressive course and high mortality, and current therapeutic options for cholangiopathies are limited. Ursodeoxycholic acid (UDCA) is the most commonly used agent for treating cholangiopathies, and several studies have reported its effects in humans and rodents [13-16]. UDCA stimulates excretion of bile acids, other organic anions (e.g., bilirubin), and $\mathrm{HCO}_{3}-$ from hepatocytes into bile canaliculi by transporter proteins. The therapeutic effects are noticeably greater during the early phase than the chronic phase [17-18]. Therefore, elucidating bile canalicular changes during the early phase is extremely important for developing a therapeutic strategy for treating cholangiopathies.

Here we explored the bile canaliculi in DDC-fed mice to characterize the morphological changes that occur during the early phase of cholangiopathy. This study will expand our understanding of the pathogenesis of cholangiopathies, and it may ultimately lead to novel therapeutic strategies.

\section{Materials and methods}




\subsection{Animals}

Various stains and genders of mice are differentially susceptible to DDC [19-20]. BALB/c mice display relatively slow progression and an intermediate degree of pathological changes [19]. Thus, to observe the morphological changes during the early phase (the first 7 days), male BALB/c mice that were 2 to 3 months old and weighing 25-30 g were used for all experiments, and age-matched BALB/c mice served as controls. To induce sclerosing cholangitis, mice were fed a diet containing $0.1 \%$ DDC (Sigma-Aldrich, St. Louis, USA) for 1, 2, 4, and 7 days. Control mice were fed a standard mouse diet. The animals were sacrificed after these times, and blood was collected from the heart. For light and electron microscopic analyses, mice were perfused for $1 \mathrm{~min}$ with saline and fixed by transcardial (about 100 mmHg) perfusion with $10 \%$ buffered formaldehyde or $2 \%$ glutaraldehyde. At least six mice were treated and analyzed per time period. All mice were housed individually in cages under specific pathogen-free conditions. All experimental protocols were approved by the local Animal Care and Use Committee, according to the criteria outlined in the Guide for the Care and Use of Laboratory Animals prepared by the National Academy of Sciences, as published by the National Institutes of Health (NIH, 1996) [21].

\subsection{Serum Biochemical Analysis}

Serum samples were stored at $-80{ }^{\circ} \mathrm{C}$ until analyses of aspartate aminotransferase (AST), 
alanine aminotransferase (ALT), and alkaline phosphatase (ALP) with a Hitachi 7180 analyzer (Hitachi, Tokyo, Japan).

\subsection{Histopathology}

Pieces of liver were perfusion fixed in $10 \%$ neutral buffered formaldehyde and embedded in paraffin. Sections were cut $4 \mu \mathrm{m}$ thick and stained with hematoxylin and eosin (H\&E), Azan, or periodic acid Schiff (PAS) for histological evaluation of liver necrosis, inflammation, fibrosis, and glycogen stores. Liver inflammatory activity represents the grade of involvement, and fibrosis represents the stage of involvement. Grading of periportal interface hepatitis and portal inflammation and staging of fibrosis were performed according to the Ishak system [22]. Quantification of cytokeratin (CK)-positive cells was performed in 10 portal tracts with portal veins measuring $50-150 \mu \mathrm{m}$ in diameter. Quantification of Ki67-positive cells was performed in 10 randomly selected bile ductules, and quantification of focal necrotic cells was performed in 10 randomly selected areas in each specimen at a magnification $\times 400$. Histology of specimens was assessed by a pathologist $(\mathrm{HK})$ who was blinded to the treatment.

\subsection{Immunohistochemical Studies}

Immunohistochemical staining of specimens was performed according to the 
manufacturer's instructions. Briefly, antigen retrieval was carried out in a pressure cooker by boiling in $10 \mathrm{mM}$ citrate buffer ( $\mathrm{pH}$ 6.0), followed by washing with phosphate-buffered saline (PBS). Endogenous peroxidase was then quenched with $3 \% \mathrm{H}_{2} \mathrm{O}_{2}$ for 10 min at room temperature. After rinsing, slides were treated overnight at $4{ }^{\circ} \mathrm{C}$ with negative control reagent or the following optimally diluted primary antibodies: carcinoembryonic antigen-related cell adhesion molecule 1 (CEACAM1; mouse monoclonal; 1:400; eBioscience, San Diego, USA), $\alpha$-smooth muscle actin ( $\alpha$ SMA; rabbit polyclonal; 1:400; Abcam, Cambridge, USA), pancytokeratin (rabbit polyclonal; prediluted; Nichirei, Tokyo, Japan), and Ki67 (mouse monoclonal; 1:100; Novocastra, Newcastle, UK). Slides were incubated with anti-mouse and anti-rabbit horseradish peroxidase-conjugated secondary antibody (goat polyclonal; prediluted; MBL, Nagoya, Japan). Binding to the antibody was detected using the labeled polymer method. Diaminobenzidine (MBL) was used as the chromagen, followed by counterstaining with hematoxylin.

\subsection{Transmission and scanning electron microscopic analyses}

Perfusion-fixed livers were cut into 1-mm sections for transmission electron microscopy (TEM) and into 2-mm sections for scanning electron microscopy (SEM). These sections were immersion fixed in $2 \%$ glutaraldehyde at $4{ }^{\circ} \mathrm{C}$ for 2 hours. The specimens for TEM were extensively washed with PBS, postfixed in $1 \%$ osmium tetroxide, dehydrated in a graded 
series of ethanol, and embedded in Epon. Ultra-thin sections $(80 \mathrm{~nm})$ were cut on an Ultra microtome EM UC6 (Leica, Vienna, Austria), stained with 1\% uranyl acetate, counterstained with the Reynolds method, and examined on an H-7650 electron microscope (Hitachi). The specimens for SEM were postfixed in $1 \%$ osmium tetroxide, dehydrated in a graded series of ethanol, and dried. The sections were then coated with a thin layer of platinum/palladium and visualized under an S-4700 electron microscope (Hitachi).

\subsection{Statistical Analysis}

Data are reported as the arithmetic means \pm SE of six animals in each group. Data were analyzed with SPSS version 20 Software (SPSS, Tokyo, Japan). The statistical significance was determined with the Mann-Whitney $U$ test with Bonferroni correction for multiple comparisons. A $P$-value $<0.05$ was considered significant.

\section{Results}

3.1. DDC feeding induces cholestasis and hepatomegaly during the early phase of sclerosing cholangitis

To elucidate the morphological and functional changes during the early phase of DDC-induced sclerosing cholangitis, we obtained tissue samples during the first 7 days of 
DDC feeding. We first performed serum biochemical and macroscopic analyses. Compared with control mice, all DDC-fed mice showed significantly increased serum AST and ALT levels (Control AST level (0 day): $206.3 \pm 46.2$; Control ALT level: $35.2 \pm 2.2$ ), indicating hepatocyte injury (Fig. 1A and B). The ALP level, an indicator of the degree of cholestasis, was significantly elevated in DDC-treated mice in a time-dependent manner (Control ALP level: $190.5 \pm 13.2$ ) (Fig. 1C). Macroscopically, even after only 1 day of DDC feeding, the skin of the ears, paws, and tail of DDC-fed mice showed yellowish coloration, indicating jaundice (data not shown). Within 7 days of DDC feeding, the liver was clearly enlarged and showed brown discoloration; the gallbladder showed dark bile and gallstones (Fig. 2A and C). Control liver and gallbladder were normal in appearance (Fig. 2A and B). The liver weight/body weight ratio ( $\mathrm{LW} / \mathrm{BW}$ ) was significantly increased in a time-dependent manner from day 2 (Control LW/BW (\%): $4.67 \pm 0.26$ ) (Fig. 2D). On the other hand, no significant difference was seen in the spleen weight/body weight ratio (SW/BW), an indicator of portal hypertension (Control SW/BW (\%): $0.46 \pm 0.03$ ) (Fig. 2E).

To further evaluate the morphological changes during the first 7 days, we next performed light microscopic, TEM, and SEM analyses. Histologically, porphyrin pigment plugs in bile canaliculi and bile ductules were observed beginning on day 1 of DDC feeding (Supplementary Fig. 1A). These plugs were also seen with TEM analysis (Supplementary Fig. 1B). Focal hepatocellular necrosis was observed beginning on day 1 and was prominent at 
days 1 and 2 compared with days 4 and 7 (Fig. 3A and B). Phagocytosis of these necrotic cells by polymorphonuclear neutrophils (PMNs) and Kupffer cells was confirmed with H\&E staining and TEM analysis (Fig. 3A, Supplementary Fig. 2A and B). PAS staining showed marked depletion of glycogen in DDC-fed mice (Supplementary Fig. 3), which was most prominent at day 1. TEM also showed reduced glycogen particles (data not shown). Immunohistochemical staining with anti-Ki67 showed significantly increased numbers of Ki67-positive hepatocytes at days 2, 4, and 7, which indicates compensatory hepatocyte proliferation (Supplementary Fig. 4). At days 2, 4, and 7, obvious inflammatory cell infiltration followed by interface hepatitis was observed in the portal area (Fig. 3A, C, and D). These inflammatory cell infiltrates were also seen with TEM and SEM (data not shown).

3.2. DDC feeding induces bile canalicular abnormalities during the early phase of sclerosing cholangitis

We hypothesized that morphological changes in bile canaliculi that are associated with hepatocellular damage appear during the early phase of cholangiopathy pathogenesis. We therefore performed CEACAM1 immunostaining to observe bile canalicular changes during the first 7 days of DDC feeding [23-24]. In control mice, anti-CEACAM1 antibody labeled bile canaliculi in the liver parenchyma, whereas the antibody did not label sinusoids (Fig. 4). The bile canaliculi demonstrated double contours, were aligned in orderly lines, and showed 
a "chicken-wire"-like appearance in control mice (Fig. 4 and Supplementary Fig. 5) [25]. In contrast, after 1 day, the bile canaliculi were dilated and showed meandering lines. This aberrant arrangement was augmented in a time-dependent manner (Fig. 4). The immunoreactivity intensity was reduced over time, and the reduction was prominent in Zone 3 (pericentral vein zone) compared with Zone 1 (periportal zone) (Supplementary Fig. 5). This difference in immunoreactivity between Zone 1 and Zone 3 may be due to susceptibility to toxic effects [26]. These bile canalicular abnormalities were also confirmed with SEM and TEM (Fig. 4). SEM and TEM analyses also revealed loss of microvilli (Fig. 4), irregular protrusions of cytoplasmic membranes, and aberrantly arranged tight junctions (Supplementary Fig. 6).

3.3. Bile canalicular abnormalities precede ductular reactions and periductal fibrosis in DDC-induced sclerosing cholangitis

Bile canalicular abnormalities such as dilatation and meandering appeared beginning on day 1 of DDC feeding as demonstrated above, whereas most previous reports state that the primary injury in cholangiopathies including PSC occurs in bile ductules that are located in the portal area. To study the timing of when the ductular reactions appear in DDC-induced sclerosing cholangitis, we performed immunohistochemical staining for cholangiocytes in bile ductules with anti-CK and anti-Ki67 antibodies. We observed no apparent change in 
morphology or numbers of bile ductules in control mice and at days 1 and 2 after DDC feeding (Fig. 5A-C). However, at days 4 and 7, ductular reactions were seen (Fig. 5A). The numbers of CK- and Ki67-positive cells at days 4 and 7 were significantly increased compared with control mice and DDC-fed mice after 1 and 2 days (Fig. 5B and C). Consistent with these findings, aSMA-positive cells (myofibroblasts) were significantly increased at days 4 and 7 (data not shown). SEM and TEM analyses also showed increased numbers of bile ductules with irregular cilia and irregular protrusions of cytoplasmic membranes (Supplementary Fig. 7A and B).

To observe the timing when periductal fibrosis appears in DDC-induced sclerosing cholangitis, we performed Azan staining. Periductal fibrosis appeared on day 7, whereas control mice and mice fed DDC for 1, 2, and 4 days showed no fibrotic changes (Fig. 3A and E). SEM and TEM analyses confirmed the increased numbers of collagen fibers in periductal areas on day 7 (data not shown).

\subsection{DDC feeding induces sinusoidal capillarization and intracellular organelle injury}

Bile canalicular (apical side of hepatocytes) abnormalities appeared earlier in DDC-induced sclerosing cholangitis than ductular reactions and periductal fibrosis. The bile canaliculi are formed by the apical membranes of hepatocytes. To further address the effects of DDC in other regions of hepatocytes, sinusoids (basal/lateral side of hepatocytes) and 
intracellular organelles were evaluated by SEM and TEM analyses. SEM and TEM showed depletion of pores in sinusoids, an increase in collagen fibers, and the deposition of debris in the space of Disse at days 4 and 7 (Fig. 6). Increased collagen fibers were not detected with Azan staining (Fig. 3A). In contrast, control mice and mice fed DDC for 1 and 2 days did not show these findings. These results indicate that sinusoidal capillarization, debris deposition, and pericellular fibrosis appeared during the first 7 days of DDC feeding. DDC-fed mice also showed increased numbers of abnormal intracellular organelles such as irregularly shaped mitochondria with fractured cristae, autophagosomes, distended Golgi apparatuses, and endoplasmic reticula with dilated cristae (Supplementary Fig. 8).

\section{Discussion}

We provide the first evidence showing that bile canalicular abnormalities appear prior to ductular reactions in a DDC-induced sclerosing cholangitis model. Using a bile duct-ligated rodent model, ductular reactions have been reported to occur early in cholangiopathy [27-30]. The bile duct-ligated rodent model seems unsuitable for studying the early phase of cholangiopathies. Rodents subjected to this treatment show rapid deterioration and development of ductular reactions within a few days. Consequently, there is not enough time to evaluate the morphological changes that occur before the ductular reactions appear. In contrast, the DDC-induced mouse model used in this study shows slow disease progression. 
This model provides sufficient time to explore the early phase of cholangiopathies and may accurately reflect the early phase of human cholangiopathies such as PSC and PBC.

For pathological diagnosis with liver specimens biopsied from patients with liver diseases, estimation of the intensity of inflammation is evaluated by examining the inflammatory cells in the portal area (portal inflammation) and the destruction of hepatocytes lining the interface between the portal area and the parenchyma (interface hepatitis) [22]. We explored portal inflammation and interface hepatitis that appeared during the first 7 days after DDC feeding and demonstrated that these inflammatory changes appeared after the morphological abnormalities in bile canaliculi. These results indicate that the observation of bile canaliculi may be useful for early diagnosis rather than observations of portal and interface regions. It is difficult for pathologists to evaluate bile canaliculi on routine H\&E sections. To evaluate the morphological changes in bile canaliculi, we used anti-CEACAM1 antibody, which is specific for bile canaliculi in the human liver [24]. CEACAM1 is one of the few CEA family members and is highly expressed in bile canalicular membranes in the mature liver of rodents and human [24, 31-32]. Our immunohistochemistry data clearly revealed that the bile canaliculi injured by DDC showed dilatation and meandering lines (Fig. 4). The anti-CEACAM1 antibody may be useful for the early diagnosis of cholangiopathies in the clinical setting.

The morphological changes observed in bile canaliculi during the early phase of 
cholangiopathies as shown in this study may lead to earlier diagnosis in clinical settings such as follow-up biopsy after liver transplantation and in familial cholangiopathies. Early diagnosis can prevent progression of cholangiopathies with the use of appropriate therapies. Recently, several therapeutic agents such as INT-767, $\beta$-adrenoceptor, 24-norUDCA, curcumin, and darbepoetin have been developed for treating cholangiopathies [33-38]. The therapeutic effects of these agents as well as UDCA are greater during the early phase than during the chronic phase. Therefore, early diagnosis by detecting the morphological changes shown in our study and the early initiation of treatments using these agents may improve the outcome in patients with cholangiopathies.

This study demonstrated some electron microscopic findings in addition to the appearance of bile canaliculi during the early phase of cholangiopathy. We observed significantly reduced numbers of pores in sinusoids, pronounced deposition of debris in the space of Disse, irregular protrusion of cytoplasmic membranes in bile ductules and bile canaliculi, and irregularly shaped cilia. The significantly reduced numbers of pores in sinusoids and the pronounced deposition of debris in the space of Disse were found with SEM and TEM analyses beginning on day 4. Furthermore, significantly increased numbers of collagen fibers in the space of Disse were found at day 7. Xie et al. also reported that capillarization precedes fibrosis in a rat model of liver cirrhosis and suggested that sinusoidal endothelial cell differentiation is an integral part of the development of fibrosis [39]. To our knowledge, no 
report has described a role for endothelial cells in the early phase of cholangiopathies. Such an observation in the DDC-induced model is a novel aspect of the early abnormalities in this disease. Lyoumi et al. observed irregular protrusions of the cytoplasmic membranes of cholangiocytes in the DDC-induced mouse model. However, no report has presented this finding in bile canaliculi (Supplementary Fig. 6A and 7B). These protrusions are likely formed in association with hepatocellular injuries, and further investigation of their pathological importance in cholangiopathies is required. The abnormal cilia in bile ductules (Supplementary Fig. 7A) have been described in previous reports studying biliary atresia, a type of congenital cholangiopathy [40-41]. Philips et al. reported that abnormalities in cilia and canaliculi appear at a very early stage of embryonic liver development, suggesting that cholestasis develops in utero. Their results are consistent with those of our study, which revealed these abnormalities during the early phase of DDC-induced sclerosing cholangitis. Therefore, further investigation is necessary to elucidate the interaction of bile canaliculi and cilia during the early phase of cholangiopathies.

We revealed that damage to various regions of hepatocytes such as the apical side (bile canaliculus), basal/lateral side (sinusoid), and intracellular organelles appeared during the first 7 days. We did not clarify the region of hepatocytes that was primarily injured by DDC. There are two possible mechanisms of damaging hepatocytes. One is the production of toxic bile acids via the hepatocytes that are stimulated by DDC. Toxic bile acids may directly 
injure the bile canaliculi, and the damage would then spread to other regions in the hepatocytes. The other possibility is direct toxicity of DDC for hepatocytes entirely. In either case, the hepatocyte is the first target in DDC-induced sclerosing cholangitis. Together with the fact that the injury to bile canaliculi constructed by hepatocytes (bile canalicular abnormalities) precedes injury to bile ductules constructed by cholangiocytes (ductular reactions), ductular reactions are deemed to be a secondary phenomenon in cholangiopathies including PSC and PBC. Our study focused on the morphological alterations of hepatocytes, especially of bile canaliculi, and cholangiocytes. These observations are necessary for elucidating the precise pathogenesis in the early phase of cholangiopathies.

In conclusion, we show that bile canalicular abnormalities precede ductular reactions in DDC-induced sclerosing cholangitis. In both human cholangiopathies such as PSC and PBC and rodent models, detailed morphological evaluation of bile canaliculi may help expand our understanding of the pathogenesis and lead to novel therapeutic strategies for cholangiopathies. 


\section{Conflict of interest statement}

The authors declare no conflict of interest.

\section{Acknowledgements}

We gratefully acknowledge Haruyasu Kohda and Keiko Furuta for their skillful technical assistance with electron microscopy. We also acknowledge Yu Kakimoto for her excellent assistance in the preparation of this paper. 


\section{References}

[1] Yamamoto K, Itoshima T, Tsuji T, et al. Three-dimensional fine structure of the biliary tract: scanning electron microscopy of biliary casts. J Electron Microsc Tech 1990;14(3):208-217.

[2] Murakami T, Sato H, Nakatani S, et al. Biliary tract of the rat as observed by scanning electron microscopy of cast samples. Arch Histol Cytol 2001;64(4):439-447.

[3] Roskams TA, Theise ND, Balabaud C, et al. Nomenclature of the finer branches of the biliary tree: canals, ductules, and ductular reactions in human livers. Hepatology 2004;39(6):1739-1745.

[4] Trauner M, Boyer JL. Bile salt transporters: molecular characterization, function, and regulation. Physiol Rev 2003;83(2):633-671.

[5] Lazaridis KN, Strazzabosco M, Larusso NF. The cholangiopathies: disorders of biliary epithelia. Gastroenterology 2004;127(5):1565-1577.

[6] Wu CT, Davis PA, Luketic VA, et al. A review of the physiological and immunological functions of biliary epithelial cells: targets for primary biliary cirrhosis, primary sclerosing cholangitis and drug-induced ductopenias. Clin Dev Immunol 2004;11(3-4):205-213.

[7] Trauner M, Fickert P, Baghdasaryan A, et al. New insights into autoimmune cholangitis through animal models. Dig Dis 2010;28(1):99-104.

[8] Trauner M, Fickert P, Halilbasic E, et al. Lessons from the toxic bile concept for the 
pathogenesis and treatment of cholestatic liver diseases. Wien Med Wochenschr 2008;158(19-20):542-548.

[9] Roskams T, Desmet V. Ductular reaction and its diagnostic significance. Semin Diagn Pathol 1998;15(4):259-269.

[10] Povero D, Busletta C, Novo E, et al. Liver fibrosis: a dynamic and potentially reversible process. Histol Histopathol 2010;25(8):1075-1091.

[11] Jiao J, Friedman SL, Aloman C. Hepatic fibrosis. Curr Opin Gastroenterol 2009;25(3):223-229.

[12] Fickert P, Stoger U, Fuchsbichler A, et al. A new xenobiotic-induced mouse model of sclerosing cholangitis and biliary fibrosis. Am J Pathol 2007;171(2):525-536.

[13] Trauner M, Graziadei IW. Review article: mechanisms of action and therapeutic applications of ursodeoxycholic acid in chronic liver diseases. Aliment Pharmacol Ther 1999;13(8):979-996.

[14] Beuers U, Boyer JL, Paumgartner G. Ursodeoxycholic acid in cholestasis: potential mechanisms of action and therapeutic applications. Hepatology 1998;28(6):1449-1453.

[15] Prieto J, Garcia N, Marti-Climent JM, et al. Assessment of biliary bicarbonate secretion in humans by positron emission tomography. Gastroenterology 1999;117(1):167-172.

[16] Fickert P, Zollner G, Fuchsbichler A, et al. Effects of ursodeoxycholic and cholic acid feeding on hepatocellular transporter expression in mouse liver. Gastroenterology 
2001;121(1):170-183.

[17] Corpechot C, Carrat F, Bonnand AM, et al. The effect of ursodeoxycholic acid therapy on liver fibrosis progression in primary biliary cirrhosis. Hepatology 2000;32(6):1196-1199.

[18] Pusl T, Beuers U. Ursodeoxycholic acid treatment of vanishing bile duct syndromes. World J Gastroenterol 2006;12(22):3487-3495.

[19] Hanada S, Strnad P, Brunt EM, et al. The genetic background modulates susceptibility to mouse liver Mallory-Denk body formation and liver injury. Hepatology 2008;48(3):943-952.

[20] Hanada S, Snider NT, Brunt EM, et al. Gender dimorphic formation of mouse Mallory-Denk bodies and the role of xenobiotic metabolism and oxidative stress. Gastroenterology 2010;138(4):1607-1617.

[21] NIH. Guide for the Care and Use of Laboratory Animals. National Academy Press: National Academy of Science, 1996, ISBN: 0-309-05377-3.

[22] Ishak K, Baptista A, Bianchi L, et al. Histological grading and staging of chronic hepatitis. J Hepatol 1995;22(6):696-699.

[23] Gu A, Zhang Z, Zhang N, et al. Generation of human CEACAM1 transgenic mice and binding of Neisseria Opa protein to their neutrophils. PLoS One 2010;5(4):e10067.

[24] Prall F, Nollau P, Neumaier M, et al. CD66a (BGP), an adhesion molecule of the carcinoembryonic antigen family, is expressed in epithelium, endothelium, and myeloid cells in a wide range of normal human tissues. J Histochem Cytochem 1996;44(1):35-41. 
[25] Arias IM, Che M, Gatmaitan Z, et al. The biology of the bile canaliculus, 1993. Hepatology 1993;17(2):318-329.

[26] Lindros KO. Zonation of cytochrome P450 expression, drug metabolism and toxicity in liver. Gen Pharmacol 1997;28(2):191-196.

[27] Mair M, Zollner G, Schneller D, et al. Signal transducer and activator of transcription 3 protects from liver injury and fibrosis in a mouse model of sclerosing cholangitis. Gastroenterology 2010;138(7):2499-2508.

[28] Fickert P, Thueringer A, Moustafa T, et al. The role of osteopontin and tumor necrosis factor alpha receptor-1 in xenobiotic-induced cholangitis and biliary fibrosis in mice. Lab Invest 2010;90(6):844-852.

[29] Polimeno L, Azzarone A, Zeng QH, et al. Cell proliferation and oncogene expression after bile duct ligation in the rat: evidence of a specific growth effect on bile duct cells. Hepatology 1995;21(4):1070-1078.

[30] Liu Z, Sakamoto T, Ezure T, et al. Interleukin-6, hepatocyte growth factor, and their receptors in biliary epithelial cells during a type I ductular reaction in mice: interactions between the periductal inflammatory and stromal cells and the biliary epithelium. Hepatology 1998;28(5):1260-1268.

[31] Gray-Owen SD, Blumberg RS. CEACAM1: contact-dependent control of immunity. Nat Rev Immunol 2006;6(6):433-446. 
[32] Sundberg U, Obrink B. CEACAM1 isoforms with different cytoplasmic domains show different localization, organization and adhesive properties in polarized epithelial cells. J Cell Sci 2002;115(Pt 6):1273-1284.

[33] Baghdasaryan A, Claudel T, Gumhold J, et al. Dual farnesoid X receptor/TGR5 agonist INT-767 reduces liver injury in the Mdr2-/- (Abcb4-/-) mouse cholangiopathy model by promoting biliary $\mathrm{HCO}(-)(3)$ output. Hepatology 2011;54(4):1303-1312.

[34] Strack I, Schulte S, Varnholt H, et al. beta-Adrenoceptor blockade in sclerosing cholangitis of Mdr2 knockout mice: antifibrotic effects in a model of nonsinusoidal fibrosis. Lab Invest 2011;91(2):252-261.

[35] Fickert P, Wagner M, Marschall HU, et al. 24-norUrsodeoxycholic acid is superior to ursodeoxycholic acid in the treatment of sclerosing cholangitis in Mdr2 (Abcb4) knockout mice. Gastroenterology 2006;130(2):465-481.

[36] Denk GU, Maitz S, Wimmer R, et al. Conjugation is essential for the anticholestatic effect of NorUrsodeoxycholic acid in taurolithocholic acid-induced cholestasis in rat liver. Hepatology 2010;52(5):1758-1768.

[37] Baghdasaryan A, Claudel T, Kosters A, et al. Curcumin improves sclerosing cholangitis in Mdr2-/- mice by inhibition of cholangiocyte inflammatory response and portal myofibroblast proliferation. Gut 2010;59(4):521-530.

[38] Sigal M, Siebert N, Zechner D, et al. Darbepoetin-alpha inhibits the perpetuation of 
necro-inflammation and delays the progression of cholestatic fibrosis in mice. Lab Invest 2010;90(10):1447-1456.

[39] Xie G, Wang X, Wang L, et al. Role of differentiation of liver sinusoidal endothelial cells in progression and regression of hepatic fibrosis in rats. Gastroenterology 2012;142(4):918-927 e916.

[40] Phillips MJ, Azuma T, Meredith SL, et al. Abnormalities in villin gene expression and canalicular microvillus structure in progressive cholestatic liver disease of childhood. Lancet 2003;362(9390):1112-1119.

[41] Chu AS, Russo PA, Wells RG. Cholangiocyte cilia are abnormal in syndromic and non-syndromic biliary atresia. Mod Pathol 2012;25(5):751-757. 
Figure legends

Fig. 1: Quantification of serum levels of (A) aspartate aminotransferase (AST), (B) alanine aminotransferase (ALT), and (C) alkaline phosphatase (ALP) in control (day 0) and 3,5-diethoxycarbonyl-1,4-dihydrocollidine (DDC)-fed mice. Data shown are expressed as the mean \pm SEM from 6 mice per group. ${ }^{\mathrm{a}} P<0.05$ compared with control mice (day 0 ). ${ }^{\mathrm{b}} P<0.05$ compared with DDC-fed mice (day 1 ). ${ }^{\mathrm{c}} P<0.05$ compared with DDC-fed mice (day 2).

Fig. 2: (A) Macroscopic appearance of livers from control mice (left) and mice fed DDC for 7 days (right). (B) Gallbladder of control mice. (C) Gallbladder of mice fed DDC for 7 days. (D) Liver weight/body weight (LW/BW) ratios of control (0 day) and DDC-fed mice. (E) Spleen weight/body weight (SW/BW) ratios of control and DDC-fed mice. Data shown are expressed as the mean \pm SEM from 6 mice per group. ${ }^{\text {a }} P<0.05$ compared with control mice (day 0). ${ }^{\mathrm{b}} P<0.05$ compared with DDC-fed mice (day 1 ). ${ }^{\mathrm{c}} P<0.05$ compared with DDC-fed mice (day 2). ${ }^{\mathrm{d}} P<0.05$ compared with DDC-fed mice (day 4). NS = not significant.

Fig. 3: (A) Microscopic appearance of livers from control and DDC-fed mice. Left panels show H\&E staining in the parenchyma. Arrowheads indicate focal necrotic hepatocytes with infiltration of polymorphonuclear neutrophils (PMNs) and Kupffer cells. (bars $=25 \mu \mathrm{m})$ Middle panels show H\&E staining in the portal area. Portal inflammation and interface 
hepatitis are seen in DDC-fed mice (days 4 and 7). Pigment plugs are found in mice fed DDC for 4 days. (bars $=25 \mu \mathrm{m}$ ) Right panels show Azan staining in the portal area. Fibrous expansion is seen in DDC-fed mice (day 7). (bars $=25 \mu \mathrm{m})(\mathrm{B})$ Histological grading of focal necrosis in control (0 day) and DDC-fed mice. (C) Histological grading of portal inflammation in control and DDC-fed mice. The bars indicate the mean histological grade of each group. (D) Histological grading of interface hepatitis in control and DDC-fed mice. (E) Histological staging of fibrosis in control and DDC-fed mice. Data shown are expressed as the mean \pm SEM from 6 mice per group. ${ }^{\mathrm{a}} P<0.05$ compared with control mice (day 0$) .{ }^{\mathrm{b}} P$ $<0.05$ compared with DDC-fed mice (day 1$) .{ }^{c} P<0.05$ compared with DDC-fed mice (day 2 ). ${ }^{\mathrm{d}} P<0.05$ compared with DDC-fed mice (day 4).

Fig. 4: The morphology of control bile canaliculi and morphological deterioration of the bile canaliculi from DDC-fed mice is shown. Column 1: Immunohistochemical staining for carcinoembryonic antigen-related cell adhesion molecule 1 (CEACAM1) (bars $=10 \mu \mathrm{m})$. Column 2: Low-magnification scanning electron microscopy (SEM) images (bars $=2 \mu \mathrm{m})$. Red arrowheads indicate bile canaliculi. Column 3: High-magnification SEM images (bars = $500 \mathrm{~nm}$ ). Column 4: Ttransmission electron microscopy (TEM) images (bars $=1 \mu \mathrm{m})$ of the bile canaliculi in control and DDC-fed mice. Red asterisks highlight the location of bile canaliculi. 
Fig. 5: (A) Immunohistochemical staining for cytokeratin (CK) (left panels) and Ki67 (right panels) in control and DDC-fed mice (bars $=50 \mu \mathrm{m}$ ). (B) Quantification of CK-positive cells in 10 portal tracts with portal veins measuring $50-150 \mu \mathrm{m}$ in diameter in control (0 day) and DDC-fed mice (left column). Quantification of Ki67-positive cells in 10 randomly selected bile ductules in control and DDC-fed mice at $\times 400$ magnification (right column). Data shown are expressed as the mean \pm SEM from 6 mice per group. ${ }^{\mathrm{a}} P<0.05$ compared with control mice (day 0). ${ }^{\mathrm{b}} P<0.05$ compared with DDC-fed mice (day 1). ${ }^{\mathrm{c}} P$ $<0.05$ compared with DDC-fed mice (day 2). ${ }^{\mathrm{d}} P<0.05$ compared with DDC-fed mice (day 4).

Fig. 6: The morphological changes in sinusoids and the space of Disse in control and DDC-fed mice (day 7) are shown. Column 1: Low-magnification SEM images (bars $=2 \mu \mathrm{m}$ ). Arrows highlight the sinusoidal space. Column 2: High-magnification SEM images (bars = $500 \mathrm{~nm}$ ). Arrowheads highlight the sinusoidal pores. Column 3: TEM images (bars $=1 \mu \mathrm{m})$. SEM shows a pronounced decrease in sinusoidal pores in DDC-fed mice (day 7). In the TEM image of DDC-fed mice, the arrow indicates debris in the space of Disse. 